\title{
Heart and Muscle in Barth Syndrome
}

\section{Josef Finsterer, MD, $\mathrm{PhD}^{1}$, Claudia Stollberger, $\mathrm{MD}^{2}$}

${ }^{1}$ Krankenanstalt Rudolfstiftung, Vienna, Austria

${ }^{2} 2$ nd Medical Dpt., Krankenanstalt Rudolfstiftung, Vienna, Austria, Europe

fifigs1@yahoo.de

Keywords: neuromuscular, cardiomyopathy, echocardiography, left ventricle, hypertrabeculation

The article by Spencer et al. on cardioskeletal findings in 34 patients with Barth-syndrome (BS), of whom half presented with left-ventricular hypertrabeculation/noncompaction (LVHT) [1], raises the following comments and concerns.

LVHT remodeled in some cases. Which was the nature of these changes? Did the number of trabeculations increase or did LVHT location/morphology change?

Concerning two brothers of whom one presented with LVHT and the other with hypertrophic cardiomyopathy, we recently observed a similar constellation in two brothers, one presenting with LVHT and the other with apical hypertrophic cardiomyopathy. These observations suggest that LVHT may be preceded by hypertrophic cardiomyopathy.

Occasionally, LVHT disappeared during follow-up. We also observed this phenomenon in a single patient. These observations suggest LVHT to develop in compensation for an insufficiently contracting myocardium [2]. In how many patients developed LVHT during follow-up?

Did endocardial fibroelastosis also affect the non-compacted layer? Was endocardial biopsy carried out in any patient? In a DMD-patient with fibrosis of the compacted layer but almost intact non-compacted layer LVHT was assumed to represent a compensatory mechanism of the failing myocardium [3].

Did those with LVHT more frequently develop ventricular arrhythmias or require an implantable cardioverter defibrilölator (ICD)?

Were relatives with BS also included? How often was LVHT found in the relatives? Was a family-screening carried out?

Which were the cardiological and neurological symptoms?

Cardiomyopathy was found in 28/31 patients. Was diastolic dysfunction detected in any of them? Did any patient present with a restrictive filling-pattern or restrictive cardiomyopathy?

Which was the indication for digitalis in 64\%? Did they have tachycardious atrial-fibrillation? Did any receive oral anticoagulation? How many required an ICD or biventricular pacemaker?

Correlated the number of ECG-abnormalities with any of the echocardiographic parameters or neurological abnormalities? Did ECG-abnormalities differ between those with/without LVHT? Was there any progression of the ECG and echocardiographic abnormalities?

Which is the pathogenetic mechanism behind hypocholesterolemia in BS [4]? Detecting the cause of hypocholesterolemia could have widespread therapeutic implications. 
Why were patients not seen by a neurologist? How many had undergone muscle biopsy? How many had a myopathy? Were hypophysial hormones determined in any of them, particularly in those with growthretardation? Were any hypophysial abnormalities found on cMRI?

There were indications for improvement of muscle force with age. Which is the pathophysiologic background of this interesting finding which could have therapeutic implications?

Overall, there is increasing evidence that LVHT is a dynamic phenomenon, remodels over time, and represents the myocardial attempt to compensate for its insufficiency from various causes.

\section{REFERENCES}

1. Spencer CT, Bryant RM, Day J, Gonzalez IL, Colan SD, Thompson WR, Berthy J, Redfearn SP, Byrne BJ. Cardiac and Clinical Phenotype in Barth Syndrome. Pediatrics 2006; (in press)

2. Finsterer J, Stollberger C, Blazek G. Neuromuscular implications in left ventricular hypertrabeculation/ noncompaction. Int J Cardiol 2006; 110: 288-300.

3. Finsterer J, Stollberger C, Feichtinger H. Noncompaction in Duchenne muscular dystrophy: frustrated attempt to create a compensatory left ventricle? Cardiology 2006; 105: 223-5.

4. Mazzocco MM, Kelley RI. Preliminary evidence for a cognitive phenotype in Barth syndrome. Am J Med Genet 2001; 102: 372-8.

Citation: Josef Finsterer, Claudia Stollberger, "Heart and Muscle in Barth Syndrome". American Research Journal of Cardiovascular Diseases, 3(1); pp: 1-2.

Copyright (C) 2019 Josef Finsterer, Claudia Stollberger, This is an open access article distributed under the Creative Commons Attribution License, which permits unrestricted use, distribution, and reproduction in any medium, provided the original work is properly cited. 